6-1-2021

\title{
Colossal intrinsic exchange bias from interfacial reconstruction in epitaxial CoFe2 04/Al2 03 thin films
}

Detian Yang

Yu Yun

Arjun Subedi

Nicholas E. Rogers

MSU Graduate Student

David M. Cornelison

Missouri State University

See next page for additional authors

Follow this and additional works at: https://bearworks.missouristate.edu/articles-cnas

\section{Recommended Citation}

Yang, Detian, Yu Yun, Arjun Subedi, Nicholas E. Rogers, David M. Cornelison, Peter A. Dowben, and Xiaoshan Xu. "Colossal intrinsic exchange bias from interfacial reconstruction in epitaxial CoFe 20 4/AI 2 03 thin films." Physical Review B 103, no. 22 (2021): 224405.

This article or document was made available through BearWorks, the institutional repository of Missouri State University. The work contained in it may be protected by copyright and require permission of the copyright holder for reuse or redistribution.

For more information, please contact BearWorks@library.missouristate.edu. 


\section{Authors}

Detian Yang, Yu Yun, Arjun Subedi, Nicholas E. Rogers, David M. Cornelison, Peter A. Dowben, and Xiaoshan Xu 


\title{
Colossal intrinsic exchange bias from interfacial reconstruction in epitaxial $\mathrm{CoFe}_{2} \mathrm{O}_{4} / \mathrm{Al}_{2} \mathrm{O}_{3}$ thin films
}

\author{
Detian Yang $₫,{ }^{1, *}$ Yu Yun $\odot,{ }^{1, *, \dagger}$ Arjun Subedi, ${ }^{1}$ Nicholas E. Rogers, ${ }^{2}$ David M. Cornelison ${ }^{\circ},{ }^{2}$ \\ Peter A. Dowben $(1),{ }^{1}$ and Xiaoshan Xu ${ }^{1,3, \ddagger}$ \\ ${ }^{1}$ Department of Physics and Astronomy, University of Nebraska, Lincoln, Nebraska 68588, USA \\ ${ }^{2}$ Department of Physics, Astronomy and Materials Science, Missouri State University, Springfield, Missouri 65897, USA \\ ${ }^{3}$ Nebraska Center for Materials and Nanoscience, University of Nebraska, Lincoln, Nebraska 68588, USA
}

(Received 30 January 2021; revised 5 May 2021; accepted 21 May 2021; published 2 June 2021)

\begin{abstract}
We have studied the epitaxial $\mathrm{CoFe}_{2} \mathrm{O}_{4}$ (111) films grown on $\mathrm{Al}_{2} \mathrm{O}_{3}$ (0001) substrates of different thickness at various temperature and discovered colossal intrinsic exchange bias up to $7 \pm 2 \mathrm{kOe}$. X-ray and electron diffraction clearly indicate an interfacial layer about $2 \mathrm{~nm}$ of different crystal structure from the "bulk" part of the $\mathrm{CoFe}_{2} \mathrm{O}_{4}$ film. The thickness dependence of the exchange bias suggests a hidden antiferromagnetic composition in the interfacial layer that couples to the ferrimagnetic "bulk" part of the $\mathrm{CoFe}_{2} \mathrm{O}_{4}$ film as the origin of the exchange bias. Considering the structural, magnetic, and electronic structure, $\mathrm{CoO}$ has been identified as the most likely candidate of the antiferromagnetic composition in the interfacial layer. This work suggests a path for enhancing intrinsic exchange bias using combination of film and substrate of large structural differences, highlighting the role of interfacial atomic and electronic reconstructions.
\end{abstract}

DOI: 10.1103/PhysRevB.103.224405

\section{INTRODUCTION}

At the interface between a ferromagnetic or ferrimagnetic (FM) and an antiferromagnetic (AFM), the exchange interaction may favor the magnetization of the FM material in a certain direction. This tendency to pin the magnetization in one direction results in a bias in the magnetic hysteresis, a phenomenon called exchange bias [1,2]. Exchange bias is fundamental to many magnetic storage and spintronic devices [2-4], and continues to be extensively studied both experimentally [2,5] and theoretically [5-7]. Although the consensus is that exchange bias originates from the pinning of magnetic moment at the FM/AFM interface, the plethora interface parameters and measurement conditions complicate the construction of a general microscopic mechanism.

A type of exchange bias, called intrinsic exchange bias, has been especially intriguing since it occurs at the interface between an FM material and a non-magnetic material, without a nominal AFM layer. Intrinsic exchange bias has been reported in a variety of heterostructures where FM thin films are epitaxially deposited on paramagnetic or diamagnetic substrates, such as $\mathrm{LaNiO}_{3} / \mathrm{LaMnO}_{3}$ superlattices [8], $\mathrm{La}_{2 / 3} \mathrm{Sr}_{1 / 3} \mathrm{MnO}_{3} / \mathrm{LaSrAlO}_{4}$ [9], $\mathrm{SrRuO}_{3} / \mathrm{LaAlO}_{3}$ [10], $\mathrm{Fe} / \mathrm{MgO}$ [11]. All proposed mechanisms suggest formation of interfacial layers with distinctly different magnetic ordering that can pin the FM magnetization. For $\mathrm{LaNiO}_{3} / \mathrm{LaMnO}_{3}$ superlattices, exchange bias comes from the induced magnetization associated with charge transfer at the interface [8]. In $\mathrm{La}_{2 / 3} \mathrm{Sr}_{1 / 3} \mathrm{MnO}_{3} / \mathrm{LaSrAlO}_{4}$, a strain-induced $\mathrm{LaSrMnO}_{4}$ based spin glass layer forms [9]. While for $\mathrm{SrRuO}_{3} / \mathrm{LaAlO}_{3}$,

\footnotetext{
${ }^{*}$ These authors contributed equally to this work.

†Corresponding author: yuyun@unl.edu

‡Corresponding author: xiaoshan.xu@unl.edu
}

an $\mathrm{AFM} \mathrm{SrRuO}$ interfacial layer is seen [10]. With Fe/MgO, $\mathrm{FeO}$ patches which form at the interface due to oxygen diffusion from the substrate, are regarded as the AFM layer. These interfacial layers are believed to pin the FM magnetization.

On the other hand, since intrinsic exchange bias relies on formation of interfacial layers, the heterostructures where intrinsic exchange bias was initially discovered [8-11], are actually not expected to have large effects, because of the film/substrate structural similarity. In other words, intrinsic exchange bias, like other emergent interfacial phenomena, is expected to be enhanced in epitaxial heterostructures of large mismatch of film/substrate structures; this is why a large intrinsic exchange bias around $2 \mathrm{kOe}$ was observed in hexagonal $\mathrm{Cr}_{2} \mathrm{Te}_{3}$ thin films deposited on a zinc-blende phase $\mathrm{CdTe}$ buffer layer [12]. In this regard, $\mathrm{CoFe}_{2} \mathrm{O}_{4}$ (CFO) thin films deposited on $\mathrm{Al}_{2} \mathrm{O}_{3}$ substrates, appear to be a promising heterostructure for achieving even larger intrinsic exchange bias.

CFO is a ferrimagnetic insulator of large magnetocrystalline anisotropy, moderate magnetization, superior mechanic hardness, and excellent physical and chemical stability, which has been widely studied $[13,14]$ and applied in high density magnetic storage [15], magnetoelectric transducers [16] and spin filters [17]. Although the face-center-cubic (fcc) inverse spinel structure of CFO shares almost no similarity with the rhombohedral corundum structure of $\mathrm{Al}_{2} \mathrm{O}_{3}$, CFO films of (111) normal direction can be epitaxially grown on $\mathrm{Al}_{2} \mathrm{O}_{3}$ (0001) substrates. The large difference in crystal structures and lattice parameter between these two materials and the large magnetoelastic effect of CFO implies the formation of interfacial layer of distinct magnetism, which is promising for large intrinsic exchange bias.

In this work, we studied crystal structures, magnetism, and electronic structure of $\mathrm{CFO}(111) / \mathrm{Al}_{2} \mathrm{O}_{3}$ (0001) thin films. Intrinsic exchange bias, as large as $7 \pm 2 \mathrm{kOe}$, has been 
observed and attributed to the interplay between the "bulk" part of the CFO film and an interfacial layer from the film reconstruction. The structural, magnetic, and electronic structural characterizations suggest $\mathrm{CoO}$, as the main AFM material, is responsible for the exchange bias at the interfacial layer.

\section{EXPERIMENTS}

\section{A. Sample preparation}

(111)-oriented CFO thin films of thicknesses from 1.7 to $55.4 \mathrm{~nm}$ were grown on $\alpha-\mathrm{Al}_{2} \mathrm{O}_{3}$ (0001) substrates by pulsed laser deposition (PLD). The KrF excimer laser of wavelength $248 \mathrm{~nm}$ was employed to ablate the CFO target with a pulse energy of $140 \mathrm{~mJ}$ and a repetition rate of $2 \mathrm{~Hz}$. The oxygen partial pressure was 10 mTorr during the growth. For all samples, the substrate temperatures were kept at $600{ }^{\circ} \mathrm{C}$ by a laser heater system during the growth. The whole growth process was in situ monitored by a reflection high energy electron diffraction (RHEED) system. After the film growth, all the samples were ex-situ annealed in one-atmosphere oxygen gas at $600{ }^{\circ} \mathrm{C}$ for 5 hours in the furnace.

\section{B. Structure characterization}

The $\theta-2 \theta$ x-ray diffraction (XRD) and $\mathrm{x}$-ray reflectivity (XRR) were conducted using a Rigaku D/Max-B x-ray diffractometer (cobalt K- $\alpha$ source, $\lambda=1.793 \AA$ ) and a Rigaku SmartLab x-ray diffractometer (copper K- $\alpha$ source, $\lambda=$ $1.5406 \AA$ ), respectively; the film thickness was extracted from the XRR data (see Fig. S1 in Supplemental Material [18]). The in-plane (IP) crystal structure was studied by analyzing time-resolved RHEED patterns recorded every 30 seconds.

\section{Magnetic characterization with SQUID}

The magnetic hysteresis loops were measured in a superconducting quantum interfere device (SQUID) system with the cooling field of $+/-70 \mathrm{kOe}$.

\section{X-ray photoelectron spectroscopy}

$\mathrm{X}$-ray photoemission spectra were acquired using VG100AX hemispherical analyzer and using a SPECS $\mathrm{X}$-ray $\mathrm{Mg} \mathrm{K}_{\alpha}$ anode $(h v=1253.6 \mathrm{eV})$ source. All the XPS measurements were carried out at room temperature in an ultra-high vacuum chamber with a base pressure lower than $3 \times 10^{-9}$ torr.

\section{RESULTS AND DISCUSSION}

\section{A. Structural characterization and evidence of interfacial reconstruction}

Since the intrinsic exchange bias typically originate from the hidden AFM interfacial layer, we carried out structural characterization of the CFO films, focusing on the film/substrate interface.

The bulk CFO has an inverse spinel crystal structure, as illustrated in Fig. 1(a). The fcc close-packed lattice of oxygen anions include two types of cation interstices: tetrahedral A sites with coordination 4 and octahedral B sites with coordination 6 . While the A sites are occupied by $\mathrm{Fe}^{3+}$, $\mathrm{B}$ sites are shared by $\mathrm{Fe}^{3+}$ and $\mathrm{Co}^{2+}$. When the CFO (111) films are grown on $\mathrm{Al}_{2} \mathrm{O}_{3}$ (0001) substrates, the significant differences between the two lattice structures are expected to cause the reconstruction at the interface in addition to the epitaxial strain in the CFO films.

The crystal structure of the CFO films was studied using $\mathrm{X}$-ray diffraction by measuring the out-of-plane (OOP) crystal spacings using $\theta-2 \theta$ scan. A representative scan for a $t=$ $22.5 \mathrm{~nm}$ sample is shown in Fig. 1(b), indicating no obvious impurity phases and the $\mathrm{CFO}(111) / \mathrm{Al}_{2} \mathrm{O}_{3}(0001)$ epitaxial relation. The spacing between the (111) planes $d_{(111)}$, calculated from the CFO (222) diffraction peaks (see Fig. S2 in Supplemental Material [18]), is displayed in Fig. 1(c) as a function of the film thickness $t$. Except for the data point at $t=3.3 \mathrm{~nm}$, $d_{(111)}$ increases to approach the bulk value [19], demonstrating that the CFO films are under a tensile strain which is released gradually as the thickness increases. The apparent outlier data point at $t=3.33 \mathrm{~nm}$ suggests the existence of an interfacial layer with a rapid change of lattice spacing. On the other hand, in the ultrathin limit, the weak intensity and large width of the diffraction peaks (see Fig. S2 in Supplemental Material [18]) make $\theta-2 \theta$ XRD a less sensitive method. Therefore, a more quantitative way of characterizing the interfacial layer is needed.

The quantitative characterization of the interfacial crystal structure of the CFO films was carried out using in-situ RHEED by measuring the in-plane (IP) diffractions. RHEED has the advantage of surface sensitivity $(\approx 1 \mathrm{~nm})$ due to the grazing incident angle. Moreover, the in-situ nature of RHEED allows the real-time measurements during the growth to reveal the evolution of crystal structure of the films as a function of thickness.

The typical RHEED images of both the CFO thin films and $\mathrm{Al}_{2} \mathrm{O}_{3}$ substrates are shown in Fig. 2(a). The electron beam was along the CFO [1110] and [1 $\overline{1} 2]$ directions for images on the left and right, respectively. The streaky patterns are consistent with the finite size of CFO grains and the 2-dimensional nature of diffraction, which indicate smooth film surfaces. Because of the fcc spinel structure of CFO, only all-odd and all-even Miller indices can survive. The IP epitaxial relation between $\mathrm{CFO}$ and $\mathrm{Al}_{2} \mathrm{O}_{3}$ can be extracted from the RHEED pattern, as depicted in Fig. 2(b), with a $30^{\circ}$ rotation between the IP reciprocal unit cell of CFO relative to that of the $\mathrm{Al}_{2} \mathrm{O}_{3}$ substrate, which is the same as that of the $\mathrm{Fe}_{3} \mathrm{O}_{4}(111) / \mathrm{Al}_{2} \mathrm{O}_{3}$ (0001) films [20].

Time-resolved RHEED was carried out to elucidate the structural evolution of the films by monitoring the top layer of the film during the growth [20]. RHEED images with electron beam along the CFO [ $1 \overline{1} 2]$ direction were taken every 30 seconds during the film growth at a repetition rate of $1 \mathrm{~Hz}$. With the growth speed $\sim 1.34 \AA / \mathrm{min}$, the deposition time can be converted to film thickness. The RHEED images were then summed up along the direction of streaks to form the RHEED spectra, i.e., RHEED intensity as a function of horizontal position. Combining all the spectra, one reaches the 2-dimensional representation of the time (or thickness) dependence of the RHEED pattern, as shown in Fig. 2(c). The CFO (-660), (-220), (2-20), (6-60) weak diffraction lines are characteristics of spinel structures, corresponding to 
(a)

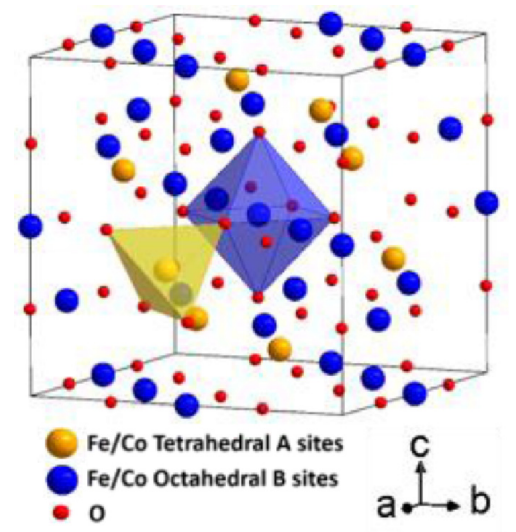

(c)

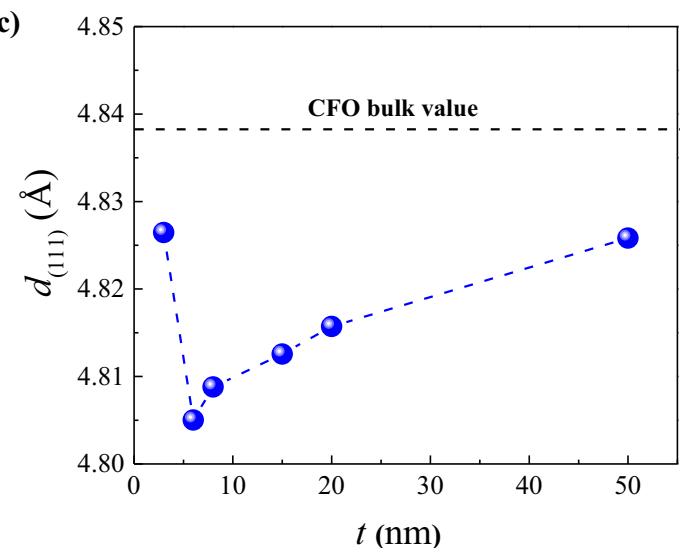

(b)

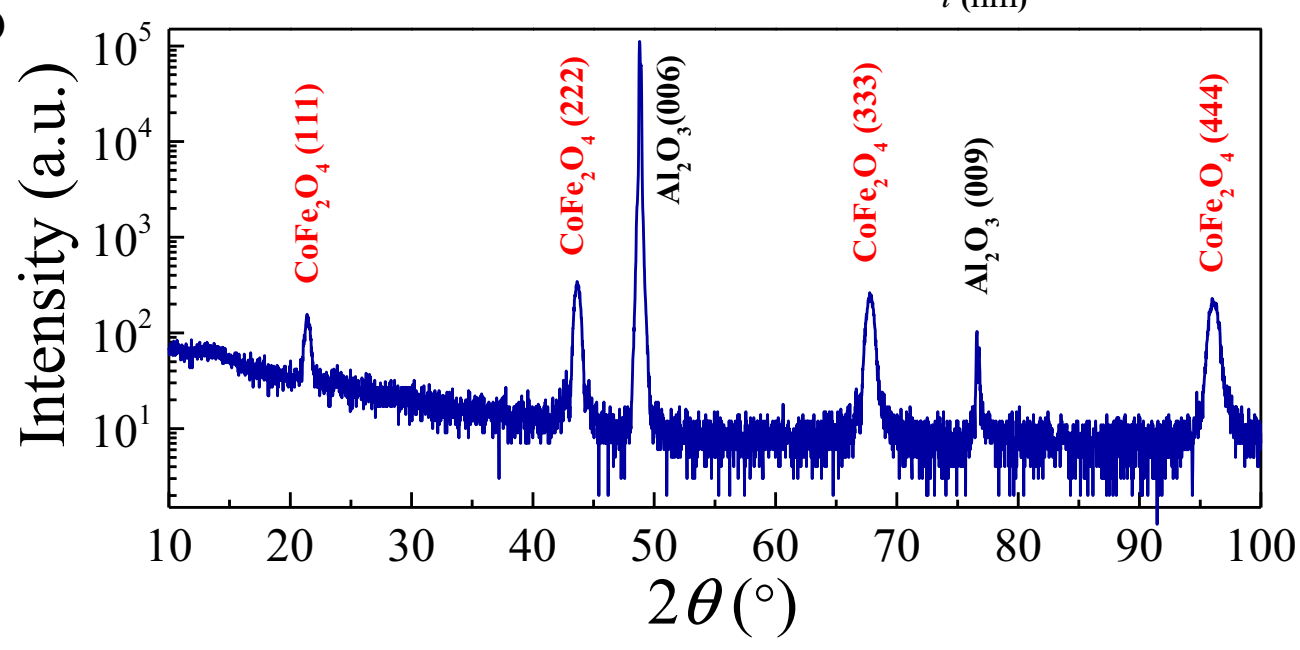

FIG. 1. Structural characterization. (a) Crystal structure of inverse spinel CFO, polyhedral model showing two interstitial sites: tetrahedral A sites marked by yellow and octahedral B sites marked by blue, respectively. (b) The $\theta-2 \theta$ XRD results of a CFO film with a thickness of $t=22.5 \mathrm{~nm}$. (c) Thickness-dependent interplane spacing of CFO (111) lattice planes, indicating a tensile strain released with thickness.

the $\mathrm{Fe} / \mathrm{Co}$ cation sublattice whose lattice constant is double of that of the fcc oxygen sublattice. Those weak lines (in the yellow dashed box) do not appear until $t \approx 2 \mathrm{~nm}$. To quantify this observation, the intensity of the weak diffraction lines was calculated as a function of the thickness; the relative IP lattice constant was also extracted from the spacings of the diffraction streaks; the results are shown in Fig. 2(d). The IP lattice constant decreases with thickness, which corroborates the tensile strain hinted in Fig. 1(c). The abrupt reduction of IP lattice constant between 1 and $2 \mathrm{~nm}$ coincides with the abrupt change in diffraction intensity of the weak lines, which is a direct evidence of existence of an interfacial layer.

To summarize the structural characterization: (1) The CFO films can be divided into an interfacial layer and the "bulk" part of the film, as illustrated in Fig. 2(e). Since the transition of lattice constant and RHEED intensity from the interface to the "bulk" in Fig. 2(d) finishes at $t \approx 2 \mathrm{~nm}$, we treat the part of film $t>2 \mathrm{~nm}$ as the "bulk" part of the film and define $t_{\mathrm{CFO} \equiv} t-t_{0}\left(t_{0}=2 \mathrm{~nm}\right)$ as its thickness. The part of film $t<2 \mathrm{~nm}$ is treated as the interfacial layer due to its dramatic differences from the "bulk" part. (2) The disappearance of the weak lines in the interfacial layer suggests the lattice constant of the interfacial layer is smaller than that of CFO, because less points in the reciprocal space means longer reciprocal base vectors and smaller separation of real-space lattice points. More specifically, if the interfacial layer also has an fcc crystal structure, which is the most likely scenario given the similar IP symmetry (see Table I in Supplemental Material [18]), the lattice constant is roughly $\frac{1}{2}$ of that of CFO, i.e., $2 a_{\text {inter }} \approx a_{\text {CFO }}$. The measured relative IP lattice constant in Fig. 2(d) further indicates that $2 a_{\text {inter }} \approx a_{\mathrm{CFO}}+0.1 \AA$.

\section{B. Magnetic characterization and colossal exchange bias}

After the structural characterizations, we measured the magnetic properties of the $\mathrm{CFO}$ films to examine the expected exchange bias and to gain more insight on the interfacial layer.

\section{Magnetic hysteresis loops containing interfacial and "bulk" components}

Figures 3(a) and 3(b) show the typical OOP and IP hysteresis loops of an $t=8.6 \mathrm{~nm}$ sample measured at $20 \mathrm{~K}$. All the hysteresis loops were measured after the samples were cooled down from room temperature under magnetic field $+/-70 \mathrm{kOe}$. Both OOP and IP hysteresis loops show the "wasp-waisted" shapes with a soft (small coercive field) and a hard (large coercive field) component, which was normally observed in CFO nanoparticles where the origin of the shape is not fully understood [21]. On the other hand, in this work, the soft and hard components can be attributed 
(a)

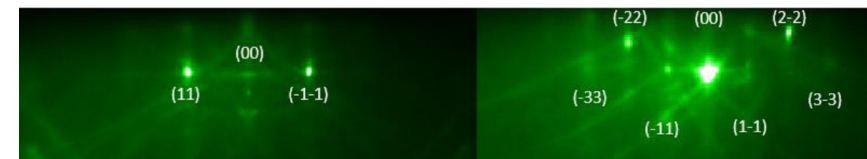

$\mathrm{Al}_{2} \mathrm{O}_{3}[1 \overline{1} 00] /(0001)$

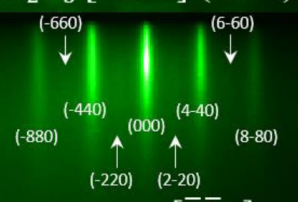

$\mathrm{CoFe}_{2} \mathrm{O}_{4}[\overline{1} \overline{1} 2]$

\section{$\mathrm{Al}_{2} \mathrm{O}_{3}[11 \overline{2} 0] /(0001)$}

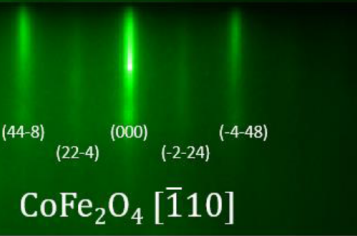

(c)
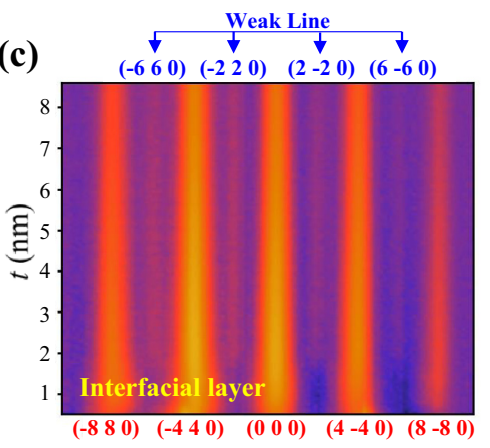

(d)

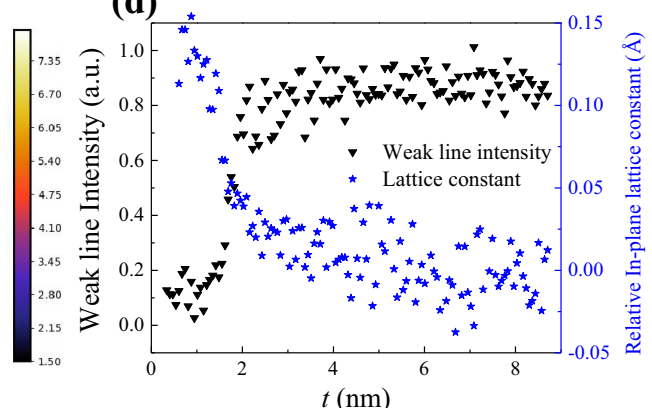

(b)

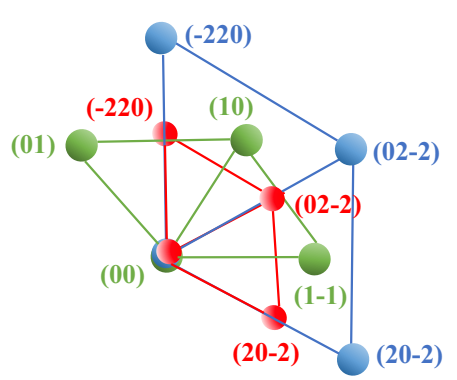

$\alpha-\mathrm{Al}_{2} \mathrm{O}_{3}$ Interfacial $\bullet \mathrm{CoFe}_{2} \mathrm{O}_{4}$

(e)

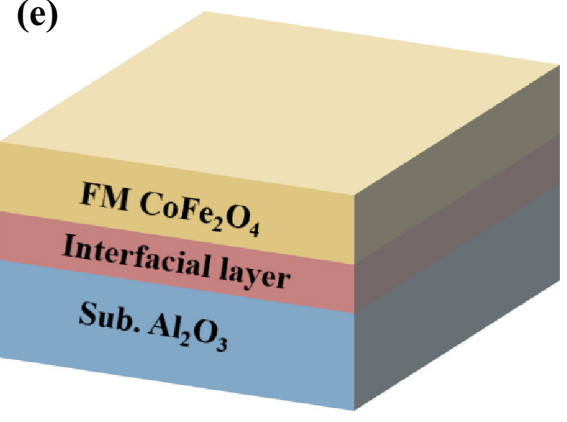

FIG. 2. Epitaxial relation of $\mathrm{CFO} / \mathrm{Al}_{2} \mathrm{O}_{3}$ films and time-resolved RHEED. (a) RHEED pattern of $\mathrm{CFO}$ films and $\mathrm{Al}_{2} \mathrm{O}_{3}$ substrates along two perpendicular IP orientations (b) IP reciprocal primitive unit cells of $\mathrm{Al}_{2} \mathrm{O}_{3}$ substrate (green), interfacial layer (blue), and $\mathrm{CoFe}_{2} \mathrm{O}_{4}(\mathrm{red})$.

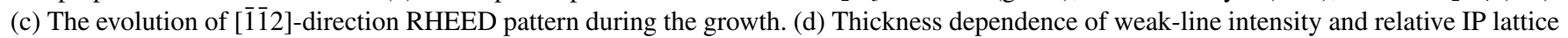
constant extracted from the RHEED patterns. (e) Schematic diagram of a structure model of the $\mathrm{CFO} / \mathrm{Al}_{2} \mathrm{O}_{3}$ heterostructures.
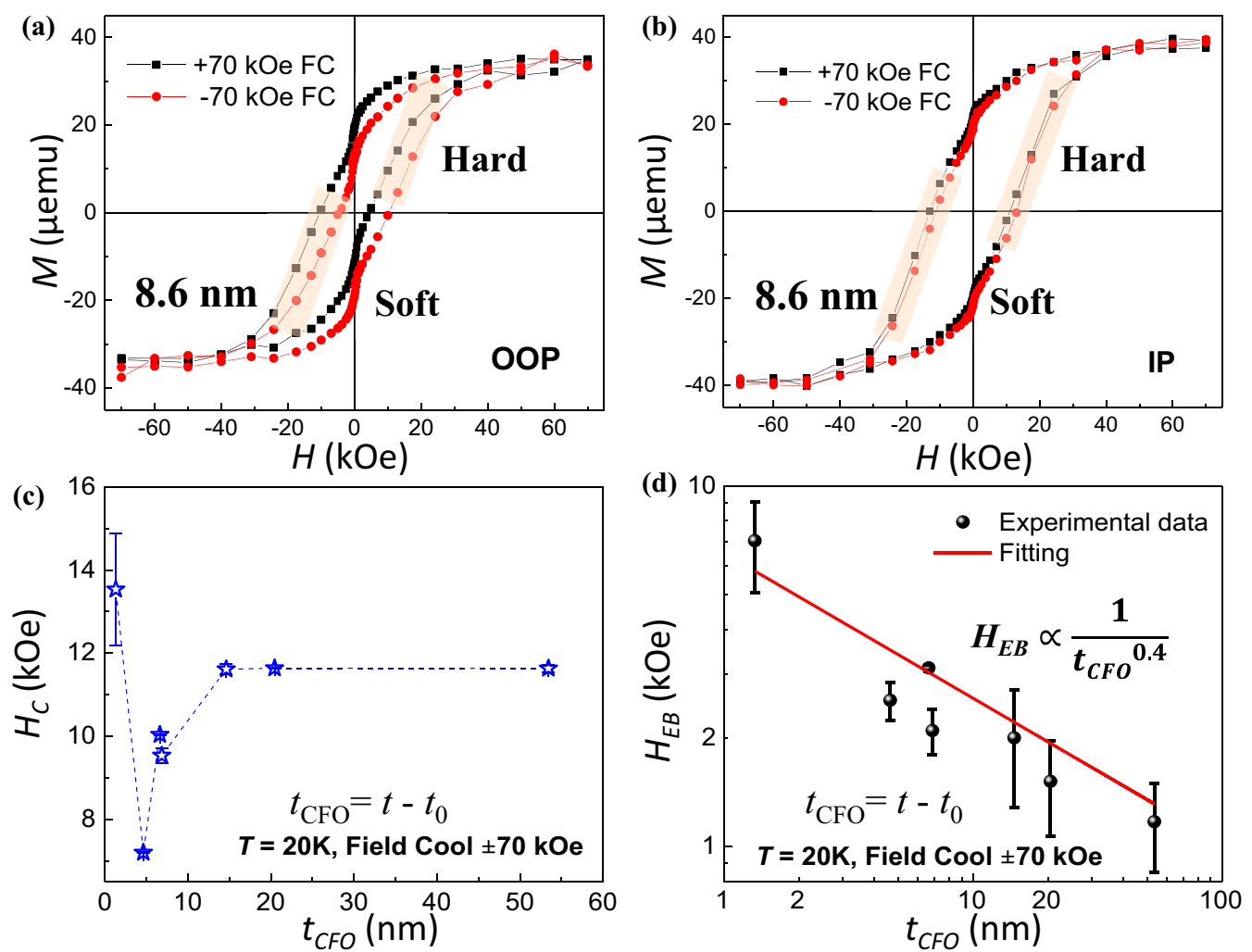

FIG. 3. IP and OOP exchange bias. Representative hysteresis loops of exchange bias with cooling magnetic field of $+/-70 \mathrm{kOe}$ along (a) OOP direction and (b) IP direction. Measuring temperature is $20 \mathrm{~K}$, and the thickness of this sample is $t=8.6 \mathrm{~nm}$. After subtracting the soft component, the OOP and IP $H_{\mathrm{EB}}$ are $3.13 \mathrm{kOe}$ and $0.89 \mathrm{kOe}$, respectively. The thickness-dependent coercivity field $\left(H_{\mathrm{C}}\right)(\mathrm{c})$ and $H_{\mathrm{EB}}(\mathrm{d})$ and at $20 \mathrm{~K}$. In (d), black balls denote the experimental data, while red line is the fitting curve. $t_{0}=2 \mathrm{~nm}$. 
to the interfacial and the "bulk" contributions since the hard component increases linearly with the film thickness while the soft component remain approximately constant when the film thickness increases (see Fig. S3 and Fig. S4 in Supplemental Material [18]).

A linear fit of the thickness dependence of the saturation magnetic moment measured at $20 \mathrm{~K}$ show that the hard component ("bulk" part of the CFO film) has a saturation magnetization $M_{\text {sat }}=1.55 \mu_{\mathrm{B}} /$ f.u., which is substantially smaller than the bulk value $3.3-3.9 \mu_{\mathrm{B}} /$ f.u. [22,23]. Reduction of magnetization of CFO film has been observed previously with several mechanisms proposed [24]. Change of ionic spin states due to strain could be a possible reason for the CFO film studied here (see discussion in the Supplemental Material Section S2.1 [18]).

The different origins of the soft and the hard components in different part of the films, also allows for extraction of the hard component by subtracting the soft component in the Supplemental Material Fig. S5 [18] (see, also references [25]) and the calculation of the average coercive field $H_{\mathrm{C}}$. The thickness dependence of $H_{\mathrm{C}}$ measured at $20 \mathrm{~K}$ is displayed as a function of $t_{\mathrm{CFO}}$ in Fig. 3(c). For $t_{\mathrm{CFO}}>5 \mathrm{~nm}, H_{\mathrm{C}}$ increases with $t_{\mathrm{CFO}}$ and reaches saturation at about $t_{\mathrm{CFO}}=14.6 \mathrm{~nm}$. This increasing trend of $H_{C}$ is consistent with previous results explained as more antiphase boundaries in thinner films [26,27]. Similar to the thickness dependence of $d_{(111)}$, the data point at $t_{\mathrm{CFO}}=1.3 \mathrm{~nm}(t=3.3 \mathrm{~nm})$ is an outlier that does not follow the overall trend, indicating a different magnetic nature of the interfacial layer.

\section{Colossal exchange bias}

Exchange bias is clearly visible in Figs. 3(a) and 3(b) as the shift of the hysteresis loops for different the field-cool (FC) conditions. An analysis of the derivative $d M / d H$ shows that the contribution of the soft component to the exchange bias is negligible, in contrast to the obvious shift of the hard component. We then calculated the exchange bias $\left(H_{\mathrm{EB}}\right)$ using the shift of the hard component of the hysteresis loops. An OOP $H_{\mathrm{EB}}=3.1 \mathrm{kOe}$ and an IP $H_{\mathrm{EB}}=0.9 \mathrm{kOe}$ were observed, respectively, at $20 \mathrm{~K}$ for the $t=8.6 \mathrm{~nm}$ film.

Thickness dependent $H_{\mathrm{EB}}$ was derived from the hysteresis loops measured at $20 \mathrm{~K}$ with OOP magnetic field. As plotted in Fig. 3(d), $H_{\mathrm{EB}}$ increases when the film thickness decreases, reaching a colossal value $=7 \pm 2 \mathrm{kOe}$ for the $t_{\mathrm{CFO}}=1.3 \mathrm{~nm}$ $(t=3.3 \mathrm{~nm})$ film. This trend of thickness dependence of $H_{\mathrm{EB}}$ is expected for an interfacial effect where the magnetization of the "bulk" part of the CFO film is pinned by an AFM interfacial layer via the exchange interaction. Furthermore, the thickness dependence of $H_{\mathrm{EB}}$ can be fitted by a power law $H_{\mathrm{EB}} \propto 1 / t_{\mathrm{CFO}}^{n}$ where $n=0.4$. In the interfacebased exchange bias models [28,29], the power-law thickness dependence with $n=1$ corresponds to a sharp and ideal interface. The power $n=0.4$ may originate from the finite transition thickness between the AFM layer and the "bulk" part of the CFO film revealed in Fig. 2(d).

Temperature dependence of $H_{\mathrm{EB}}$ derived from the hysteresis loops measured for the $t=8.6 \mathrm{~nm}\left(t_{\mathrm{CFO}}=6.6 \mathrm{~nm}\right)$ CFO film is plotted in Figs. 4(a) and 4(b) for OOP and IP magnetic field, respectively. For both the OOP and IP fields and $T>25 \mathrm{~K}, H_{\mathrm{EB}}$ decreases with temperature and disappears at around $T=250 \mathrm{~K}$. Since $M_{\text {sat }}$ stays approximately at $1.5 \mu_{\mathrm{B}} /$ f.u. as $T>25 \mathrm{~K}$ for both the OOP and IP magnetic field [Fig. 4(c) and 4(d)], the reduction of $H_{\mathrm{EB}}$ at higher temperature can be understood as the reduction of the interfacial exchange energy $E_{\mathrm{ex}}$ that needs to be compensated by $H_{\mathrm{EB}}$ as $E_{\mathrm{ex}}=H_{\mathrm{EB}} M_{\mathrm{sat}}$, where $M_{\mathrm{sat}}$ is the saturation magnetization. When temperature increases, $E_{\mathrm{ex}}$ is expected to decrease and eventually vanish at the ordering temperature of the AFM layer. Therefore, the ordering temperature of the AFM layer is above or close to $250 \mathrm{~K}$. Below $T=25 \mathrm{~K}, H_{\mathrm{EB}}$ increases with temperature, which can be attributed to the rapid decrease of $M_{\text {sat }}$ from $4.5 \mu_{\mathrm{B}} /$ f.u. to $1.5 \mu_{\mathrm{B}} / \mathrm{f}$.u., in the Supplemental Material Section S2.3 (see, also, reference [30,31])

Summarizing the magnetic characterization, a colossal exchange bias up to $H_{\mathrm{EB}}=7 \pm 2 \mathrm{kOe}$ for the $t=3.3 \mathrm{~nm}$ $\left(t_{\mathrm{CFO}}=1.3 \mathrm{~nm}\right) \mathrm{CFO}$ film at $20 \mathrm{~K}$ has been observed. The thickness dependence of $H_{\mathrm{EB}}$ suggests that the magnetization of the "bulk" part of the CFO film is pinned by the interaction with the interfacial layer, suggesting the interfacial layer most likely has an AFM ordering since the exchange bias survives under the $70 \mathrm{kOe}$ field. The temperature dependence of the $H_{\mathrm{EB}}$ hints that the AFM ordering temperature is above and close to $250 \mathrm{~K}$. In addition, the interfacial layer also exhibits ferromagnetic behavior with a small coercive field.

\section{Electronic structural characterization and confirmation of interfacial layer}

To further elucidate the nature of the interfacial layer, we studied the electronic structure of $\mathrm{CFO}(111) / \mathrm{Al}_{2} \mathrm{O}_{3}(0001)$ films using $\mathrm{x}$-ray photoelectron spectroscopy (XPS). The XPS Co $2 \mathrm{p}_{3 / 2}$ core level spectra for CFO films of thicknesses $t=5.5 \mathrm{~nm}$ and $t=1.7 \mathrm{~nm}$ grown on $\mathrm{Al}_{2} \mathrm{O}_{3}$ are depicted in Figs. 5(a) and 5(c), respectively. For the $t=5.5 \mathrm{~nm}$ film, the Co $2 \mathrm{p}_{3 / 2}$ XPS core level spectra contain three components: $\mathrm{P}_{1}$ at $781.4 \mathrm{eV}, \mathrm{P}_{2}$ at $783.7 \mathrm{eV}$, and $\mathrm{S}$ (satellite) at $788.1 \mathrm{eV}$. For the $t=1.7 \mathrm{~nm}$ film, these three Co $2 \mathrm{p}_{3 / 2}$ core level features, $\mathrm{P}_{1}, \mathrm{P}_{2}$ and $\mathrm{S}$ are at the smaller binding energies of $781.0 \mathrm{eV}$, $783.4 \mathrm{eV}$, and $786.5 \mathrm{eV}$, respectively.

As illustrated in Fig. 5, for both the $1.7 \mathrm{~nm}$ and the $5.5 \mathrm{~nm}$ films, the binding energies of $\mathrm{P}_{1}, \mathrm{P}_{2}$, and $\mathrm{S} C \mathrm{Co} 2 \mathrm{p}_{3 / 2}$ core level components are somewhat greater than the CFO film Co $2 \mathrm{p}_{3 / 2}$ core level component binding energies of $780.4 \mathrm{eV}, 782.8 \mathrm{eV}$ and $786.2 \mathrm{eV}$ [32], $779.8 \mathrm{eV}, 781.9$ and $785.9 \mathrm{eV}$ [33] and $779.8 \mathrm{eV}, 781.4$ and $785.5 \mathrm{eV}$ [34] reported previously. These somewhat larger binding energies are consistent with a dielectric CFO grown on a dielectric substrate, although less than the binding energies of $787.0 \mathrm{eV}, 789.4 \mathrm{eV}$ and $793.4 \mathrm{eV}$ reported elsewhere [35]. For the $1.7 \mathrm{~nm}$ film, the binding energies of some of these three Co $2 p_{3 / 2}$ core level features are in agreement with the results reported by Wan and Li [36]. Although the spectrum for Co $2 \mathrm{p}_{3 / 2}$ core level features in the work of Wan and $\mathrm{Li}$ [36] lacks a peak equivalent to $\mathrm{P}_{2}$ in our work, the binding energy values of spectral components $P_{1}$ and $\mathrm{S}$ in our work for a $1.7 \mathrm{~nm}$ thick film are in agreement with their values of $780.9 \mathrm{eV}$ and $785.5 \mathrm{eV}$, respectively. The value of binding energy for the Co $2 \mathrm{p}_{3 / 2}$ core level feature (S) seems to be higher in our work, than in the work of Wan and 

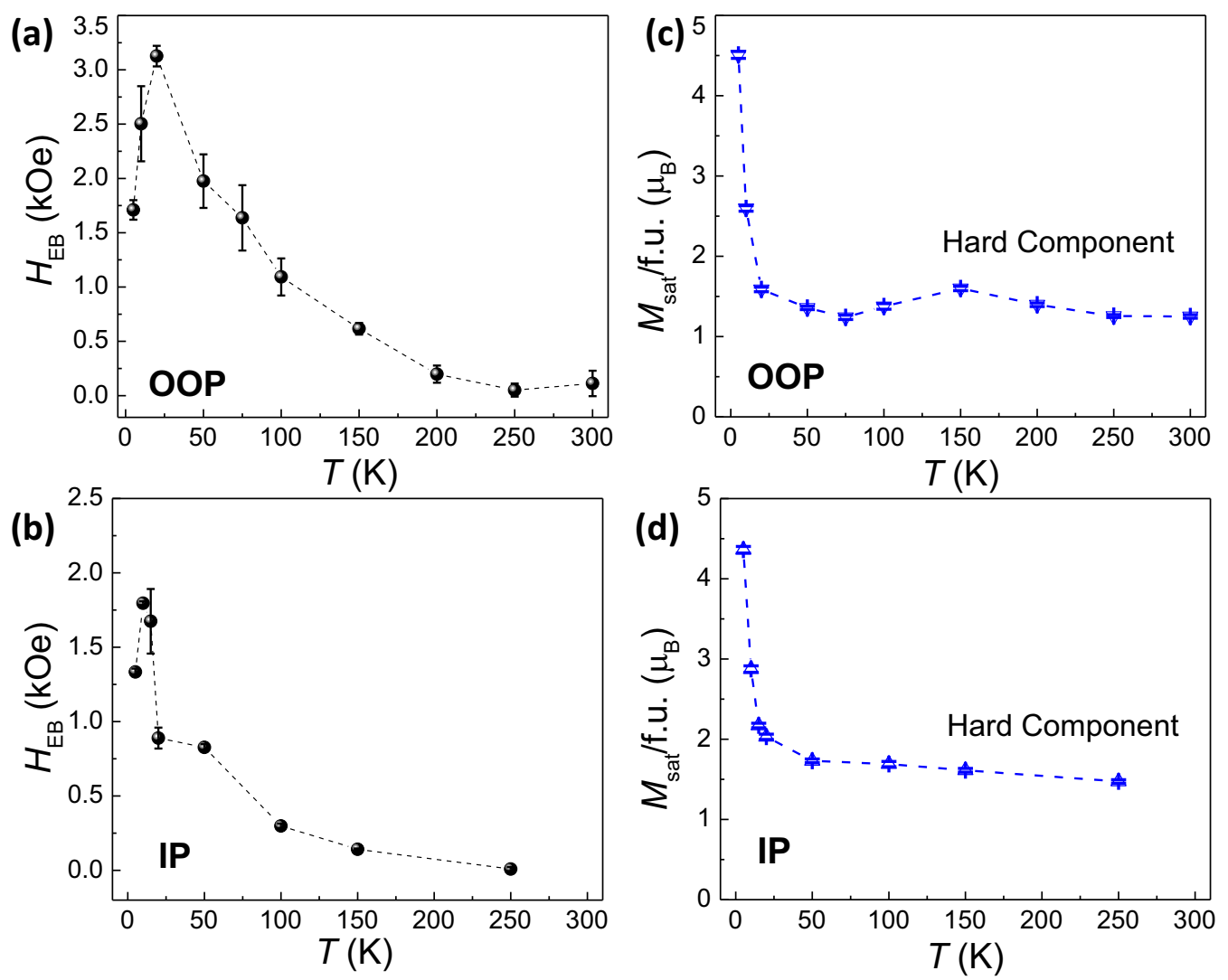

FIG. 4. Temperature-dependent exchange bias and saturation magnetization for the CFO thin film of $t=8.6 \mathrm{~nm}\left(t_{\mathrm{CFO}}=6.6 \mathrm{~nm}\right) . H_{\mathrm{EB}}$ measured in OOP (a) and IP (b) fields. Saturation magnetization $M_{\text {Sat }}$ measured in OOP (c) and IP (d) fields.

$\mathrm{Li}$ [36] but this could be a result of a poor fitting of the Co $2 \mathrm{p}_{3 / 2}$ spectrum in the latter work.

These three Co $2 p_{3 / 2}$ core level features, labeled $\mathrm{P}_{1}, \mathrm{P}_{2}$, and $\mathrm{S}$ in Fig. 5, are typically assigned to the cobalt placed in the cation octahedral and tetrahedral sites [32-35], respectively, as well as an additional Co $2 \mathrm{p}_{3 / 2}$ core level satellite feature at even larger binding energy. The cation tetrahedral and octahedral sites are the A and B sites of Fig. 1(a). This assignment cation octahedral and tetrahedral sites fails here because these two components differ substantially in surface weight. This is evident in the changing ratio of $\mathrm{P}_{1}$ to $\mathrm{P}_{2}$ with emission angle, obtained from angle-resolved XPS on the $5.5 \mathrm{~nm}$ film as plotted in Fig. 5(b), since XPS becomes more surface sensitive as the photoelectron emission angle, with respect to the surface normal, increases [37-39]. The higher ratio of $\mathrm{P}_{1} / \mathrm{P}_{2}$ Co $2 \mathrm{p}_{3 / 2}$ XPS spectra components, at higher emission angles, show that more $\mathrm{P}_{1}$ species is present at the surface than $\mathrm{P}_{2}$, thereby making $\mathrm{P}_{1}$ the surface core level and $\mathrm{P}_{2}$ the bulk core level components of Co $2 \mathrm{p}_{3 / 2}$ core level. Such a change in $\mathrm{P}_{1} / \mathrm{P}_{2}$ ratio is consistent with a surface with a cobalt species that differs from the bulk and calls into question the assignments of the octahedral $\mathrm{O}_{\mathrm{h}}$ and tetrahedral $\mathrm{T}_{\mathrm{d}}$ site occupancy based on the core level photoemission intensities, as done elsewhere $[32,33,35]$. The $\mathrm{P}_{1}$ and $\mathrm{P}_{2}$ core level features in the Co $2 \mathrm{p}_{3 / 2}$ XPS spectra for the $5.5 \mathrm{~nm}$ film and that of the $1.7 \mathrm{~nm}$ film are tantamount to a surface to bulk core level shift, but there must be recognition that the surface oxide may differ substantially from the bulk oxide. The resulting surface-to-bulk core level shift in the core level binding energy is ascribed here to the different chemical environments of the Co cations at the surface compared to the Co atom in the bulk part of the $5.5 \mathrm{~nm}$ film [38-41].

The core level photoemission satellite feature (feature $S$ ) at 6 to $7 \mathrm{eV}$ greater binding energy has been observed in both the $1.7 \mathrm{~nm}$ and the $5.5 \mathrm{~nm}$ films, consistent with a 2-hole bound state common to CFO [32-36,42-44] and cobalt oxides with a band gap [45-49]. Similar satellite peaks have been commonly observed in oxides with $\mathrm{Co}^{2+}$ such as $\mathrm{CFO}$ and $\mathrm{CoO}$, but not as obvious in $\mathrm{Co}_{3} \mathrm{O}_{4}$ in which $\mathrm{Co}^{3+}$ dominates [45,49,50].

In addition to the binding energy shifts in the Co $2 \mathrm{p}_{3 / 2}$ core level photoemission components, observed between $5.5-\mathrm{nm}$ and 1.7-nm thick films, the 1.7-nm-thick film has an additional peak $\left(\mathrm{P}_{0}\right)$ with $779.4 \mathrm{eV}$ binding energy, changing the shape of Co $2 p_{3 / 2}$ core level spectra at the lower binding energies. This additional Co $2 \mathrm{p}_{3 / 2}$ core level binding energy component, $\mathrm{P}_{0}$, has a larger binding energy than $778.3 \mathrm{eV}$ [51] to $778.1 \mathrm{eV}$ [46], the Co $2 p_{3 / 2}$ core level binding energy of cobalt metal [51] and is indicative of a reduced oxide in the Co $2 \mathrm{p}_{3 / 2}$ core level spectrum in the $1.7 \mathrm{~nm}$ film, similar to $\mathrm{CoO}$ at a binding energy of 779.7 [45] and $780[46,49]$. Since the $1.7 \mathrm{~nm}$ film is close to the $\sim 2 \mathrm{~nm}$ interfacial AFM layer discovered from the structural characterization, the electronic structure of this film is expected to reflect the properties of the $\mathrm{CFO} / \mathrm{Al}_{2} \mathrm{O}_{3}$ interface. This additional $\mathrm{P}_{0}$ Co $2 \mathrm{p}_{3 / 2}$ XPS spectra component, in the thinner CFO films, supports the 

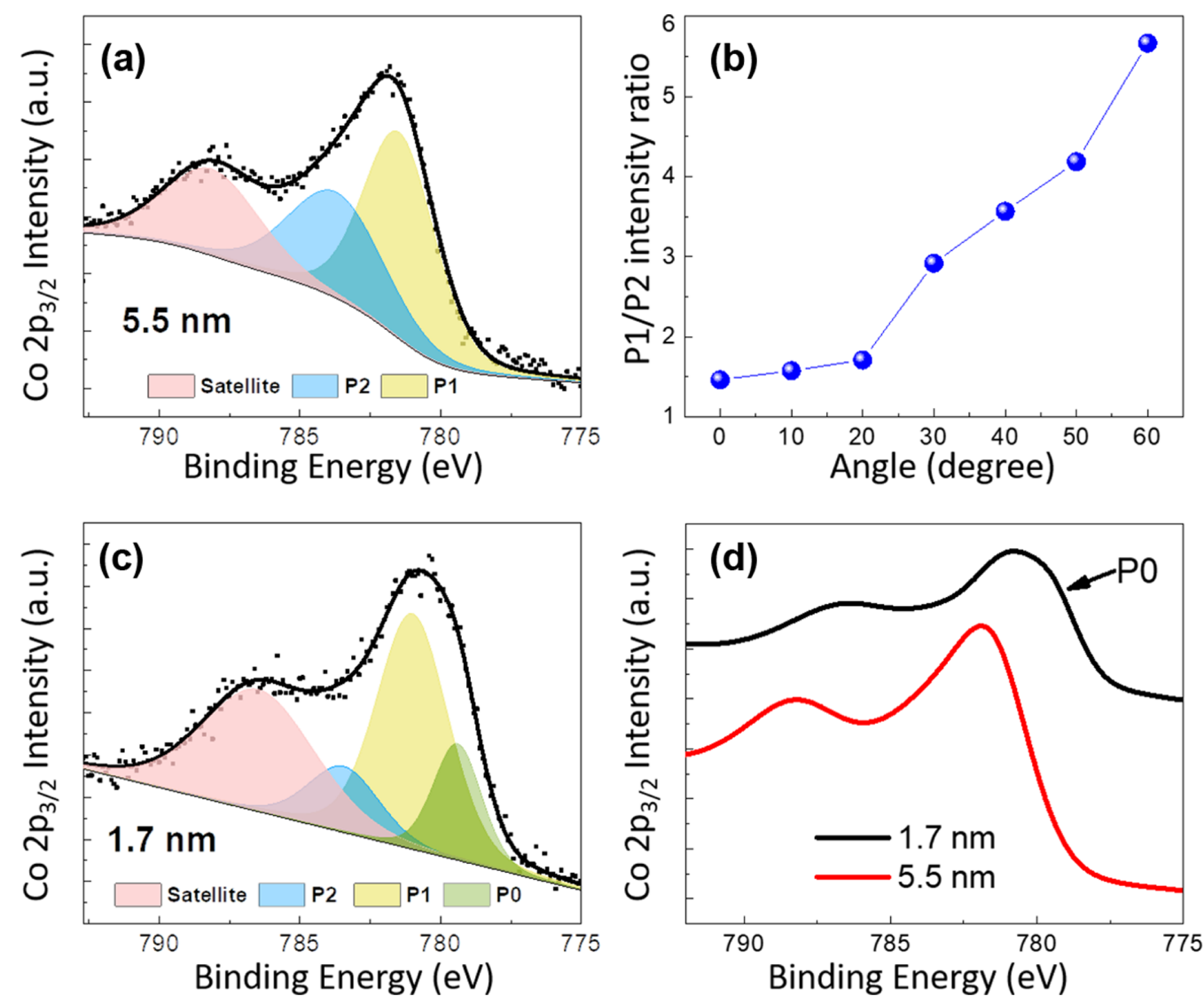

FIG. 5. XPS of $\mathrm{CFO} / \mathrm{Al}_{2} \mathrm{O}_{3}$ films. The XPS of the Co $2 \mathrm{p}_{3 / 2}$ core level features in $\mathrm{CFO} / \mathrm{Al}_{2} \mathrm{O}_{3}$ with the CFO film of thicknesses (a) $t=5.5 \mathrm{~nm}$ and (c) $t=1.7 \mathrm{~nm}$. The Co $2 \mathrm{p}_{3 / 2}$ core level photoemission spectrum, for the $t=5.5 \mathrm{~nm}$ film (a), contains three peaks: $\mathrm{P}_{1}, \mathrm{P}_{2}$, and Satellite $(\mathrm{S})$. An additional peak $\left(\mathrm{P}_{0}\right)$ on the lower binding energy side to $\mathrm{P}_{1}$ is observed in the $t=1.7 \mathrm{~nm}$ film (c). (b) The $\mathrm{P}_{1} / \mathrm{P}_{2} \mathrm{XPS}$ component intensity ratios for the Co $2 \mathrm{p}_{3 / 2}$ core level, from the $5.5 \mathrm{~nm}$ film, are plotted as a function of the photoemission take-off angle with respect to the surface normal. (d) The fitted spectra of Co $2 \mathrm{p}_{3 / 2}$ core levels for the $5.5 \mathrm{~nm}$ and $1.7 \mathrm{~nm}$ films, displaying clear evidence of the dielectric nature of the thicker $\mathrm{CFO}$ films and of an additional component peak $\left(\mathrm{P}_{0}\right)$ in the thinner films.

scenario where the Co electronic structure in the interfacial layer is different from the "bulk" part of the film. In addition, the smaller binding energies overall and the larger number of Co $2 \mathrm{p}_{3 / 2}$ XPS spectral components is indicative of suboxide $\mathrm{Co}_{x} \mathrm{O}(x>1)$ formation. In contrast, there are no additional peaks for the $\mathrm{Fe} 2 \mathrm{p}_{3 / 2}$ for CFO films with thicknesses of $5.5 \mathrm{~nm}$ and $1.7 \mathrm{~nm}$ (see Supplemental Material Fig. S7 [18]).

\section{Discussion on the nature of the interfacial layer}

According to the structural characterization, the lattice constant of the interfacial layer follows $2 a_{\text {inter }} \approx a_{\mathrm{CFO}}+$ $0.1 \AA$. A survey of the possible cobalt iron oxides (see Table S1 in Supplemental Material [18]) shows that fcc $\mathrm{FeO}$ and $\mathrm{CoO}$ whose lattice constants are $a_{\mathrm{FeO}}=4.31 \AA$ and $a_{\mathrm{CoO}}=4.26 \AA$, respectively, are reasonable candidates. Both $\mathrm{FeO}$ and $\mathrm{CoO}$ exhibit AFM order, which is consistent with the exchange bias observed in the CFO films. On the other hand, exchange bias persists up to $250 \mathrm{~K}$ [Fig. 4], which supports the key role of $\mathrm{CoO}$ since its bulk Néel temperature is about $290 \mathrm{~K}$ [52], and suggests that $\mathrm{FeO}$ is less likely because its Neel temperature is only $190 \mathrm{~K}$ [53]. The spinel $\mathrm{Co}_{3} \mathrm{O}_{4}$ is also unlikely due to the $40 \mathrm{~K}$ Neel temperature [54]. Previous work shows that the Néel temperature of a $2 \mathrm{~nm} \mathrm{CoO}$ layer may reduce to $250 \mathrm{~K}$ [55], which may be related to the observed temperature at which $H_{\mathrm{EB}}$ vanishes in Fig. 4. The OOP $H_{\mathrm{EB}}$ observed in Fig. 3(d) is also in line with the $H_{\mathrm{EB}}=3.7 \mathrm{kOe}$ reported in the CFO-CoO core-shell nanoparticles [56], suggesting the interfacial similarity between two systems. The $\mathrm{CoO}$ layer is also consistent with the electronic structure of the interfacial layer observed by XPS.

While most likely $\mathrm{CoO}$ is the hidden AFM material in the interfacial layer responsible for the exchange bias, from the stoichiometry point of view, certain form of iron oxide is expected in the interfacial layer too. Here we propose that the iron oxide takes the form $\mathrm{Fe}_{3-x} \mathrm{O}_{4}(0<x<1 / 3)$ with the (inverse-) spinel structure. The two end members of this material sequence are $\mathrm{Fe}_{3} \mathrm{O}_{4}$ and $\gamma-\mathrm{Fe}_{2} \mathrm{O}_{3}$, with bulk lattice constants $a_{\mathrm{Fe} 304}=8.39 \AA$ and $a_{\gamma-\mathrm{Fe} 303}=8.33 \AA$ respectively [57] (see Table S1 in Supplemental Material [18]), which are compatible to lattice constants of $\mathrm{CoO}$ and $\mathrm{CFO}$. Specifically, the crystal structure $\gamma-\mathrm{Fe}_{2} \mathrm{O}_{3}$ is essentially that of $\mathrm{Fe}_{3} \mathrm{O}_{4}$ with $\mathrm{Fe}$ vacancies [57]. The RHEED pattern of $\gamma$ $\mathrm{Fe}_{2} \mathrm{O}_{3}$ is also expected to have vanishing weak streaks due to the disorder [20]. Both $\mathrm{Fe}_{3} \mathrm{O}_{4}$ and $\gamma-\mathrm{Fe}_{2} \mathrm{O}_{3}$ are ferrimagnetic with high Curie temperature $\left(860 \mathrm{~K}\right.$ and $950 \mathrm{~K}$ for $\mathrm{Fe}_{3} \mathrm{O}_{4}$ and $\gamma-\mathrm{Fe}_{2} \mathrm{O}_{3}$ respectively) [58].The coercivity of the $\mathrm{Fe}_{3} \mathrm{O}_{4}$ and $\gamma-\mathrm{Fe}_{2} \mathrm{O}_{3}$ films is expected to be on the order of 100 Oe [20], consistent with the soft component observed in the hysteresis 
loop that was attributed to the interfacial layer. Essentially, the cobalt iron oxides reconstruct into separated Co-rich ( $\mathrm{CoO}$ ) and $\mathrm{Fe}$-rich $\left(\mathrm{Fe}_{3-\mathrm{x}} \mathrm{O}_{4}\right)$ phases under the large tensile strain, presumably because both $\mathrm{CoO}$ and $\mathrm{Fe}_{3-\mathrm{x}} \mathrm{O}_{4}$ can have larger lattice constants.

Although the composition $\mathrm{CoO}$ and $\mathrm{Fe}_{3-\mathrm{x}} \mathrm{O}_{4}$ can account for most of the observed structural and magnetic properties, one should be reminded of the complexity of the system. For example, the significant reduction of $M_{\text {sat }}$ of CFO and the rapid increase of $M_{\mathrm{sat}}$ at low temperature suggest the structural sensitivity of the Co spin states (see discussion in Supplemental Materials Section S2.3 [18]). In addition, the interfacial layer contains a finite transition length of lattice constants [Fig. 2(d)], which smears the boundary between the "bulk" part of the CFO film and the AFM CoO. The complex structural and magnetic properties in the transition region may contribute to the colossal $H_{\mathrm{EB}}=7.0 \mathrm{kOe}$ measured under the OOP field [Fig. 3(d)] in the $t=3.3 \mathrm{~nm}$ film. Elucidation of the microscopic mechanisms will be important for understanding and further enhancing the intrinsic exchange bias.

\section{CONCLUSIONS}

Colossal exchange bias has been observed in CFO (111)/ $\mathrm{Al}_{2} \mathrm{O}_{3}(0001)$ films, up to $H_{\mathrm{EB}}=7 \pm 2 \mathrm{kOe}$ in film of $t=$ $3.3 \mathrm{~nm}$ at $20 \mathrm{~K}$. An interfacial layer of about $2 \mathrm{~nm}$ due to reconstruction of CFO has been observed by the structure characterizations, consistent with the thickness dependence of the $H_{\mathrm{EB}}$ which indicates an exchange coupling between an AFM layer and the FM CFO layer. Considering the lattice constant and the distinct Co electronic structure of the interfacial layer, and the temperature at which $H_{\mathrm{EB}}$ vanishes, $\mathrm{CoO}$ has been identified as the AFM material in the interfacial layer responsible for biasing the magnetic hysteresis of the "bulk" part of the CFO film via exchange coupling. The magnetic hysteresis of small coercivity of the interfacial layer was attributed to the spinel $\mathrm{Fe}_{3-\mathrm{x}} \mathrm{O}_{4}$ to compensate the stoichiometry. Although many aspects of the complex heterostructure still need to be understood, this work demonstrates that interfacial reconstruction, which may be designed using film-substrate combination, is an effective way in tuning and enhancing intrinsic exchange bias.

\section{ACKNOWLEDGMENTS}

This work was primarily supported by the National Science Foundation (NSF), Division of Materials Research (DMR) under Grant No. DMR-1454618. The research was performed in part in the Nebraska Nanoscale Facility: National Nanotechnology Coordinated Infrastructure and the Nebraska Center for Materials and Nanoscience, which are supported by the NSF under Grant No. ECCS- 2025298, and the Nebraska Research Initiative.
[1] W. H. Meiklejohn and C. P. Bean, Phys. Rev. 102, 1413 (1956).

[2] J. Nogués and I. K. Schuller, J. Magn. Magn. Mater. 192, 203 (1999).

[3] J. F. Bobo, L. Gabillet, and M. Bibes, J. Condens. Matter Phys. 16, S471 (2004).

[4] B. G. Park, J. Wunderlich, X. Marti, V. Holy, Y. Kurosaki, M. Yamada, H. Yamamoto, A. Nishide, J. Hayakawa, H. Takahashi et al., Nat. Mater. 10, 347 (2011).

[5] A. E. Berkowitz and K. Takano, J. Magn. Magn. Mater. 200, 552 (1999).

[6] R. L. Stamps, J. Phys. D. Appl. Phys. 33, R247 (2000).

[7] M. Kiwi, J. Magn. Magn. Mater. 234, 584 (2001).

[8] M. Gibert, P. Zubko, R. Scherwitzl, J. Iniguez, and J. M. Triscone, Nat. Mater. 11, 195 (2012).

[9] B. Cui, C. Song, G. Y. Wang, H. J. Mao, F. Zeng, and F. Pan, Sci. Rep. 3, 2542 (2013).

[10] C. Sow, A. K. Pramanik, and P. S. Anil Kumar, J. Appl. Phys. 116, 194310 (2014).

[11] Y. Fan, K. J. Smith, G. Lupke, A. T. Hanbicki, R. Goswami, C. H. Li, H. B. Zhao, and B. T. Jonker, Nat. Nanotechnol. 8, 438 (2013).

[12] L. Hui, S. T. Lim, J. F. Bi, and K. L. Teo, J. Appl. Phys. 111, $07 \mathrm{D} 719$ (2012).

[13] S. Jauhar, J. Kaur, A. Goyal, and S. Singhal, RSC Adv. 6, 97694 (2016).

[14] F. Sharifianjazi, M. Moradi, N. Parvin, A. Nemati, A. Jafari Rad, N. Sheysi, A. Abouchenari, A. Mohammadi, S. Karbasi, Z. Ahmadi et al., Ceram. Int. 46, 18391 (2020).

[15] V. Pillai and D. O. Shah, J. Magn. Magn. Mater. 163, 243 (1996).
[16] V. L. de Brito, S. A. Cunha, L. V. Lemos, and C. B. Nunes, Sensors 12, 10086 (2012).

[17] A. V. Ramos, T. S. Santos, G. X. Miao, M. J. Guittet, J. B. Moussy, and J. S. Moodera, Phys. Rev. B 78, 180402(R) (2008).

[18] See Supplemental Material at http://link.aps.org/supplemental/ 10.1103/PhysRevB.103.224405 for additional information for structural characterization, magnetic characterization, and XPS studies.

[19] T. A. S. Ferreira, J. C. Waerenborgh, M. H. R. M. Mendonça M. R. Nunes, and F. M. Costa, Solid State Sci. 5, 383 (2003).

[20] X. Zhang, S. Yang, Z. Yang, and X. Xu, J. Appl. Phys. 120, 085313 (2016).

[21] T. Magno de Lima Alves, B. F. Amorim, M. A. Morales Torres, C. G. Bezerra, S. Nóbrega de Medeiros, P. L. Gastelois, L. E. Fernandez Outon, and A. A. M. Waldemar, RSC Adv. 7, 22187 (2017).

[22] E. W. Gorter, Philips Res. Rep. 9, 295 (1954).

[23] M. Tachiki, Prog. Theor. Phys. 23, 1055 (1960).

[24] F. Eskandari, S. B. Porter, M. Venkatesan, P. Kameli, K. Rode, and J. M. D. Coey, Phys. Rev. Mater. 1, 074413 (2017).

[25] V. A. M. Brabers and A. D. D. Broemme, J. Magn. Magn. Mater. 104-107, 405 (1992).

[26] J.-B. Moussy, S. Gota, A. Bataille, M.-J. Guittet, M. GautierSoyer, F. Delille, B. Dieny, F. Ott, T. D. Doan, P. Warin, P. Bayle-Guillemaud, C. Gatel, and E. Snoeck, Phys. Rev. B 70, 174448 (2004).

[27] A. V. Ramos, J. B. Moussy, M. J. Guittet, M. Gautier-Soyer, C. Gatel, P. Bayle-Guillemaud, B. Warot-Fonrose, and E. Snoeck, Phys. Rev. B 75, 224421 (2007).

[28] A. P. Malozemoff, J. Appl. Phys. 63, 3874 (1988). 
[29] D. V. Dimitrov, S. Zhang, J. Q. Xiao, G. C. Hadjipanayis, and C. Prados, Phys. Rev. B 58, 12090 (1998).

[30] S. Q. Antonio and M. Pompei, in 2015 AEIT International Annual Conference (AEIT) (IEEE, Piscataway, NJ, 2015), pp. 1-6.

[31] J. B. Goodenough and A. L. Loeb, Phys. Rev. 98, 391 (1955).

[32] M. Fantauzzi, F. Secci, M. Sanna Angotzi, C. Passiu, C. Cannas, and A. Rossi, RSC Adv. 9, 19171 (2019).

[33] W. P. Wang, H. Yang, T. Xian, and J. L. Jiang, Mater. Trans. 53, 1586 (2012).

[34] Z. P. Zhou, Y. Zhang, Z. Y. Wang, W. Wei, W. F. Tang, J. Shi, and R. Xiong, Appl. Surf. Sci. 254, 6972 (2008).

[35] T. Aghavnian, J. B. Moussy, D. Stanescu, R. Belkhou, N. Jedrecy, H. Magnan, P. Ohresser, M. A. Arrio, P. Sainctavit, and A. Barbier, J. Electron Spectros. Relat. Phenomena 202, 16 (2015).

[36] C. Wan and J. Li, Carbohydr. Polym. 134, 144 (2015).

[37] A. Dhingra, P. V. Galiy, L. Wang, N. S. Vorobeva, A. Lipatov, A. Torres, T. M. Nenchuk, S. J. Gilbert, A. Sinitskii, A. J. Yost et al.,, Semicond. Sci. Technol. 35, 065009 (2020).

[38] W. F. Egelhoff, Surf. Sci. Rep. 6, 253 (1987).

[39] P. A. Dowben and A. Miller, Surface Segregation Phenomena, (CRC Press, Boca Raton, 1990).

[40] D. Spanjaard, C. Guillot, M. C. Desjonquères, G. Tréglia, and J. Lecante, Surf. Sci. Rep. 5, 1 (1985).

[41] A. Rosengren and B. Johansson, Phys. Rev. B 23, 3852 (1981).

[42] M. Sánchez-Arenillas, M. Oujja, F. Moutinho, J. de la Figuera, M. V. Cañamares, A. Quesada, M. Castillejo, and J. F. Marco, Appl. Surf. Sci. 470, 917 (2019).

[43] Y. Huang, W. Yang, Y. Yu, and S. Hao, J. Electroanal. Chem. 840, 409 (2019).

[44] W. Bian, Z. Yang, P. Strasser, and R. Yang, J. Power Sources 250, 196 (2014).
[45] Y. Lykhach, S. Piccinin, T. Skala, M. Bertram, N. Tsud, O. Brummel, M. Farnesi Camellone, K. Beranova, A. Neitzel, S. Fabris et al., J. Phys. Chem. Lett. 10, 6129 (2019).

[46] M. C. Biesinger, B. P. Payne, A. P. Grosvenor, L. W. M. Lau, A. R. Gerson, and R. S. C. Smart, Appl. Surf. Sci. 257, 2717 (2011).

[47] T. J. Chuang, C. R. Bridle, and D. W. Rice, Surf. Sci. 59, 413 (1976).

[48] O. Olanipekun, C. Ladewig, J. A. Kelber, M. D. Randle, J. Nathawat, C.-P. Kwan, J. P. Bird, P. Chakraborti, P. A. Dowben, T. Cheng et al., Semicond. Sci. Technol. 32, 095011 (2017).

[49] Z.-X. Shen, J. W. Allen, P. A. P Lindberg, D. S. Dessau, B. O. Wells, A. Borg, W. Ellis, J. S. Kang, S.-J. Oh, I. Lindau, and W. E. Spicer, Phys. Rev. B 42, 1817 (1990).

[50] J. L. Ortiz-Quinonez, U. Pal, and M. S. Villanueva, ACS Omega 3, 14986 (2018).

[51] J. F. Moulder, W. F. Stickle, P. E. Sobol, and D. Bombem, Handbook of X-ray Photoeletron spectroscopy (Perkin-Elmer, Eden Prairie, 1992).

[52] F. J. Romero, J. Jiménez, and J. Del Cerro, J. Magn. Magn. Mater. 280, 257 (2004).

[53] B. D. Cullity and C. D. Graham, Introduction to Magnetic Materials (2 ed.) (Wiley-IEEE Press, NJ, 2008).

[54] W. L. Roth, J. Phys. Chem. Solids 25, 1 (1964).

[55] Y. J. Tang, D. J. Smith, B. L. Zink, F. Hellman, and A. E. Berkowitz, Phys. Rev. B 67, 054408 (2003).

[56] N. Flores-Martinez, G. Franceschin, T. Gaudisson, P. Beaunier, N. Yaacoub, J.-M. Grenèche, R. Valenzuela, and S. Ammar, Part. Part. Syst. Charact. 35, 1800290 (2018).

[57] M. Coduri, P. Masala, L. D. Bianco, F. Spizzo, D. Ceresoli, C. Castellano, S. Cappelli, C. Oliva, S. Checchia, M. Allieta et al., Nanomaterials 10, 867 (2020).

[58] H. Shokrollahi, J. Magn. Magn. Mater. 426, 74 (2017). 